\title{
Maintenance Strategy for the Road Infrastructure for the Autonomous Vehicle
}

\author{
Ikram Najeh \\ Institut VEDECOM, 23 bis allée des Marronniers, 78000 Versailles France, ikram.najeh@vedecom.fr
}

Laurent Bouillaut *, Dimitri Daucher**

Université Gustave Effeil, Grettia (IFSTTAR), F-77455 Marne-la-Vallée, France, laurent.bouillaut@ifsttar.fr Université Gustave Effeil, Lepsis (IFSTTAR), F-77455 Marne-la-Vallée, France, dimitri.daucher@ifsttar.fr

Maxime Redondin

Institut VEDECOM, 23 bis allée des Marronniers, 78000 Versailles France, maxime.redondin@vedecom.fr

\begin{abstract}
The quality of the road infrastructure plays a major role in the road safety, especially for the autonomous vehicles (AV). The AV contains cameras and lidars able to detect the road markings, obstacles and the other vehicles. The road markings help the AV to identify the path runway and to understand their localization. Thus, the AV must interact with the road infrastructure to drive around without any human interactions with a high automatization level. The failures of the components of the road infrastructure (pavement and road markings) are incompatible phenomena with the operation of $\mathrm{AV}$. To ensure a good evolution of this kind of vehicles, the infrastructure and the vehicles must coordinate, each providing a certain level of service. Thus, an efficient road maintenance must be considered. The proposed paper suggests a maintenance policy for the road infrastructure by grouping the maintenance strategies of the road markings and the pavement. This strategy considers the road infrastructure as serial system. A genetic algorithm is used to group the maintenance activities. This methodology is applied to feedback datasets from both the French National Road 4 and the American pavement using the Long-Term Pavement Performance (LTPP) database.
\end{abstract}

Keywords: Grouping maintenance actions, Road infrastructure maintenance, Autonomous vehicle, Pavement cracks, Road marking degradation

\section{Introduction}

If autonomous vehicles are considered as a potential economic, social and environmentally friendly solution to some of our current mobility needs, their operation is strongly correlated with maintaining a high level of quality of the infrastructure. Indeed, the erasure of the road marking, the appearance of longitudinal and transverse cracks are incompatible phenomena with the operation of autonomous vehicles. Those infrastructure failures can be responsible for some bias in the estimation of the distance between objects, vehicles stability and users' comfort. Moreover, the higher automatization level will be, the stronger expectations on the availability of the road infrastructure components will be.

The figure (1) represents an example of failure of the infrastructure in an autonomous vehicle context. Here, the vehicle must detect the road marking to identify the runway. In this case, the right-side marking (marked in red) is detected by the vehicle when the central line (indicated by the dot yellow line) is not. Then, the autonomous vehicle considers this road as a single-lane road. From this example we conclude that an efficient infrastructure maintenance should be considered to optimize the operation.

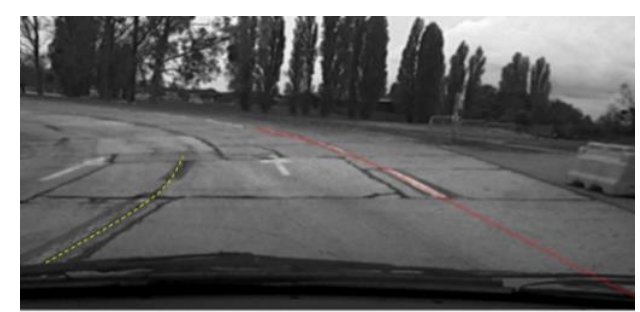

Fig. 1. Example of a disturbed detection due to a severely degraded infrastructure (Revilloud et al 2016 b)

In the same context, M. Zouch et al (2009) proposed a maintenance strategy for the longitudinal cracks to minimize the total maintenance cost. The degradation model was

Proceedings of the 30th European Safety and Reliability Conference and the 15th Probabilistic Safety Assessment and Management Conference.

Edited by Piero Baraldi, Francesco Di Maio and Enrico Zio Copyright $(92020$ by ESREL2020 PSAM 15 Organizers. Published by Research Publishing, Singapore ISBN: 981-973-0000-00-0 :: doi: 10.3850/981-973-0000-00-0 esrel2020psam15-paper 
presented as a two-phases process. During the initial phase the inferior road layers are deteriorating even if the surface is free of cracks. The first deterioration step leads to the propagation phase during which the cracks are observable, and the deterioration of inferior road layers continues. For the road marking, $M$. Redondin et al (2019) proposed two maintenance strategies using the feedback data of the French National Road 4 (NR4): the first one systematic according to the age and the second one conditional according the degradation level.

In order to propose an efficient preventive maintenance policy, the road infrastructure is considered as a multi-component system. It consists on the road markings (emergency line, center line and median strip line) and the pavement. Each component has its own maintenance strategy and the aim of this paper is to propose a grouping maintenance policy. The failure of one component makes the system is failed. Thus, the road infrastructure is presented as a serial system with 4 components.

In the literature, H.VU et al (2014) proposed a maintenance policy for systems with a complex structure using the Genetic Algorithm (GA). This strategy is about taking advantage of the maintenance action on one component to maintain other components and thus, limit the logistic consequences and optimize the maintenance organization. In the same context, Faddoul et al (2018) proposed a grouping maintenance strategy for the serial systems as an extension of the individual maintenance.

Then in this paper, the considered datasets and their preprocessing will be first introduced. Then, the clustering process of the considered infrastructure, based on the degradation model, will be detailed.

Finally, before some conclusions and prospects, maintenance actions will be optimized firstly with an individual approach and the considering a grouping strategy.

\section{Introducing of the considered databases and pre-processing}

At present, we have not identified any accessible database containing degradation monitoring for both marking and pavement, which are the two components considered in this study. It was therefore necessary to consider different database for each component. However, the approach introduced could easily be implemented on a single database the day such a feedback database will be available.

\subsection{Road marking}

Retroreflective marking has the particularity of reflecting the light emitted from the headlights of a vehicle back to the driver. A marking with a high level of retro-reflection indicates that it is white and perfectly visible. The level of retro-reflection decreases over time and the marking turns grey. A road marking must ensure a minimum level of retro-reflection of $150 \mathrm{mcd} / \mathrm{m}^{2} / \mathrm{lx}$. This threshold is proposed by M. Redondin et al (2017) and can be modified in order to optimize the detection of markings by the autonomous vehicles. Thus, the road marking is considered as failed when the retro-reflection level is less than $150 \mathrm{mcd} / \mathrm{m}^{2} / \mathrm{lx}$.

In this work, the feedback data of the French National Road 4 (RN4), that relies Paris to Strasbourg, is used. This road is inspected every September and the road markings are replaced in the following March.

\subsection{Pavement}

Long Term Pavement Performance (LTPP) is a program founded by the Federal Highway Administration (FHWA) since 1988. It collects the data of the quality of the pavement performance from test sections across the United States and Canada.

An initial analysis of this database shows that the state of Texas is the one with the most information on the percent of pavement cracking. In this work, Texas data were therefore considered for our study.

It is assumed that the Texas sections were inspected periodically every two years between 1991 and 2001 and the decision parameter is the longitudinal cracks percentage.

A section is down if the percent of cracks is greater than $5 \%$. This threshold can be modified if more information is available on the expectations of the autonomous vehicle with respect to the infrastructure.

\subsection{Censored data}

In all this study, we unfortunately have to face with censored date. Indeed, in this case of study, the road infrastructure is periodically inspected, thus the exact time of the failure (or the observed degradation state) is unknown.

Thus, when the failure happens before the first inspection, the data is left censored. In case the failure happens between two inspections $r$ and $l$ the data is interval censored. Finally, when the road infrastructure is still in good condition after the last inspection, the monitoring data are right censored.

To deal with this kind of data, McLachlan et al (2007) proposed to use the EM algorithm to estimate the date of failure. M Redondin et al (2018) proposed an extension of this algorithm for highly censored data. 


\subsection{The considered EM algorithm for censured} data estimation

In this paper, the Weibull distribution $W(\alpha, \beta)$ is defined by its associated probability density function given by equation (1) where $\alpha$ and $\beta$ are respectively the scale and shape parameters:

$$
f(x)=\left\{\begin{array}{rlrl}
\frac{\beta}{\alpha}\left(\frac{t}{\alpha}\right)^{\beta-1} & e^{\left(\frac{t}{\alpha}\right)^{\beta},} & & t>0 \\
0, & t & \leq 0
\end{array}\right.
$$

In order to estimate the parameters of a Weibull density, the EM algorithm is used to find the maximum likelihood. In this work, the observed data $\left(t_{1}, t_{2}, \ldots, t_{n}\right)$ is independent identically distributed. Redondin et al (2018) proposed a censor detector is associated for each observation as follows: $\forall i=1, \ldots, n$

$$
\delta_{i}=\left\{\begin{array}{l}
0 \text { if } t_{i} \text { is uncensored } \\
1 \text { if } t_{i} \text { is left censored } \\
2 \text { if } t_{i} \text { is interval-censored } \\
3 \text { if } t_{i} \text { is right censored }
\end{array}\right.
$$

From now, the observed data is defined by the detector as defined:

$$
\begin{aligned}
\tau & =\left\{t \in\left(\left(t_{1}, \delta_{1}\right), \ldots,\left(t_{n}, \delta_{n}\right)\right) / \delta_{i}=0\right\} \\
X & =\left\{x \in\left(\left(t_{1}, \delta_{1}\right), \ldots,\left(t_{n}, \delta_{n}\right)\right) / \delta_{i}=1\right\} \\
Y & =\left\{y \in\left(\left(t_{1}, \delta_{1}\right), \ldots,\left(t_{n}, \delta_{n}\right)\right) / \delta_{i}=2\right\} \\
Z & =\left\{z \in\left(\left(t_{1}, \delta_{1}\right), \ldots,\left(t_{n}, \delta_{n}\right)\right) / \delta_{i}=3\right\}
\end{aligned}
$$

In this case, the maximum likelihood is defined as follow:

$$
\mathrm{L}(\alpha, \beta)=\prod_{t \in \tau} f(t) \prod_{x \in \bar{X}} F(x) \prod_{y \in Y} R(r)-R(l) \prod_{z \in \bar{Z}} R(z)
$$

As detailed in McLachlan et al (2007), the EM algorithm is a two-phases process:

- The Expected Step estimates censored data according to a given Weibull distribution $W(\alpha, \beta)$.

- The Maximization Step estimates a Weibull distribution $W(\alpha, \beta)$ by the Maximum Likelihood Estimator according to both uncensored and completed data.

The EM algorithm is an iterative process. The initial Weibull distribution $W\left(\alpha_{0}, \beta_{0}\right)$ is given arbitrary. In each iteration $k$, an estimation of $\alpha_{k}$ and $\beta_{k}$ is given. The algorithm is stopped when $\left|\beta_{k}-\beta_{k-1}\right|<10^{-4}$.

\section{Clustering}

To simplify the process, the data are classified according to their degradation model. In data mining, clustering is the act and the process of grouping objects into classes (clusters). Each cluster contains objects that have similar characteristics. Many algorithms are available in the literature such as: k-means, Mean-shift clustering, Hierarchical clustering and MultiLayer clustering.

During his works, M. Redondin et al (2018) suggested to classify markings into 9 clusters using Hierarchical clustering. The same clustering methodology is used to classify the pavement sections.

\subsection{Hierarchical clustering}

In literature, there are two types of hierarchical clustering: Divisive and Agglomerative according to Sasirekha, K., \& Baby, P (2013).

In this work, the agglomerative method is used. Each reference point or pavement section is considered as an individual cluster. In each iteration, the clusters with the same characteristics (cracks percentage or the retro reflexion level) merge together in order to create a new cluster. The process is still working until having one cluster that group all individuals. To calculate the similarity between two clusters, there are certain approaches which are used to calculate the distance such as Min, Max and Ward.

In this case study, the Ward distance defined by Eq (8) in where $X$ and $Y$ are both clusters, $x$ and $y$ their average profiles, $n_{x}, n_{y}$ their numbers.

$$
d(X, Y)=\frac{d(x, y)}{\frac{1}{n_{x}}+\frac{1}{n_{y}}}
$$

The hierarchical clustering technique can be visualized using a Dendrogram. Based on its shape, the number of clusters is defined using the semi-partial $R^{2}$ criterion. It calculates the loss of between-class inertial. The loss must be as small as possible. 


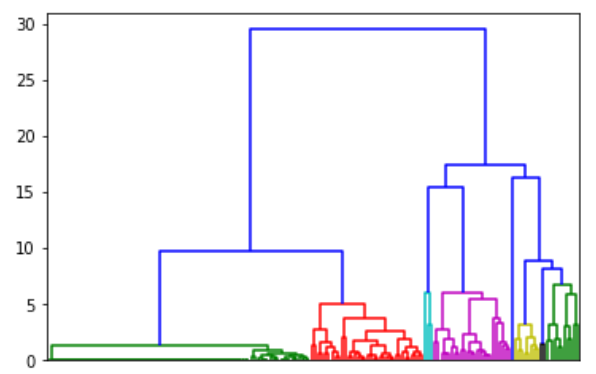

Fig. 2. Dendrogram associated to the AHC of the pavement sections

The figure 2 represents the dendrogram the pavement sections. Based on it, the pavement section can be grouped into 8 clusters. The number of clusters is defined using the semipartial criterion.

Table 1: Weibull analysis of the cluster 1 of pavement sections.

\begin{tabular}{lll}
\hline $\begin{array}{l}\text { Age Failure } \\
\text { estimated }\end{array}$ & $\begin{array}{l}\text { Interval } \\
\text { Censored }\end{array}$ & $\begin{array}{l}\text { Right } \\
\text { Censored }\end{array}$ \\
\hline 48 & $2(1,88 \%)$ & 0 \\
72 & $7(6,16 \%)$ & 0 \\
96 & $17(16,03 \%)$ & 0 \\
120 & $7(6,16 \%)$ & $73(68,8 \%)$ \\
\hline
\end{tabular}

As illustration, let's consider an example of a cluster that contains 106 sections. The table 1 represents the result of EM algorithm. It indicates that $(68,6 \%)$ of the data is right censored and $(30 \%)$ are interval censored. Particularly, the main failure is between 72 and 120 months the data are right censored.

\section{Individual maintenance strategy optimization}

The reference points of the road markings are clustered into $\mathrm{n}$ groups and the pavement sections are clustered into $\mathrm{m}$ groups. For each cluster $i$, a Weibull analysis is applied to identify the parameters $\alpha_{i}$ and $\beta_{i}$.

In this section, an age-based maintenance strategy is presented. This strategy consists in identifying a path $T^{*}$ of preventive maintenance time for each component. Based on the Weibull parameters, the preventive maintenance cost $C_{p}$ and the corrective maintenance cost $C_{c}$, the critical operational age $x_{i}{ }^{*}$ is defined. The average maintenance cost per unit of time over an infinite horizon defined in the $\mathrm{Eq}(9)$ is calculated as given by Rausand $\mathrm{M}$ and $\mathrm{A}$ Hoyland (2004). The optimum path of maintenance is defined as showed in the equation (10). If a failure happens between two planning maintenance actions, a corrective maintenance is performed, and the age of the component is initialled on 0 .

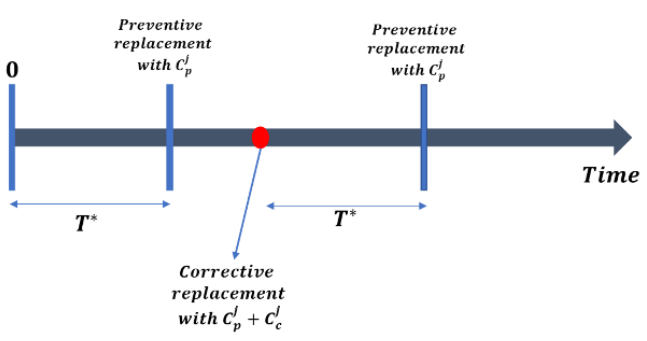

Fig 3. Example of age-based maintenance replacement

The figure (3) illustrates the age replacement maintenance strategy for a component $i$.

$$
\begin{gathered}
C_{j}(t)=\frac{C_{p}^{j}+C_{c}^{j} F_{j}(t)}{\int_{0}^{t} R_{j}(x) d x} \\
T^{*}=\inf \left\{T / C(t)=\min _{t>0} C(t)\right\}
\end{gathered}
$$

\section{Grouping maintenance actions}

This section is devoted to proposing a grouping maintenance policy. A multi-component system is a structured combination of components or subsystems that interact in an organized manner to accomplish a common functionality. In this paper, a maintenance grouping strategy for multicomponent systems is proposed.

The proposed strategy considers the structure of the system. For systems with complex structure, the components are classified into 2 types: critical if the its shutdown leads to the shutdown of the system and non-critical if the system is still functioning even if the component fails.

Let's consider $\pi_{i}$ an indicator function defined as:

$$
\pi_{i}=\left\{\begin{array}{l}
1 \text { if the component } i \text { is critical } \\
0 \text { if the component } i \text { is not }
\end{array}\right.
$$

\subsection{Planning horizon}

To group the maintenance activities, a planning horizon must be defined. It begins at $T_{B e g i n}$ and ends at $T_{E n d}$. To guarantee that all the components are maintained at least once time, $\mathrm{H}$. $\mathrm{Vu}$ et al (2014) proposed to define the $T_{E n d}$ as follows: 


$$
T_{\text {End }}=\min _{i=1, \ldots, n} t_{i}^{1}
$$

Where $t_{i}{ }^{1}$ represents the date of the first maintenance action of the component $i$, where age $e_{i}^{0}$ the age of the component $i$ at $T_{\text {Begin }}$.

As an illustration, the figure 4 is an example of a system with 4 components. The first maintenance execution time of the component 2 is the biggest, thus, in this case the planning horizon is defined by $T=\left[T_{\text {Begin }}, t_{2}^{1}\right]$.

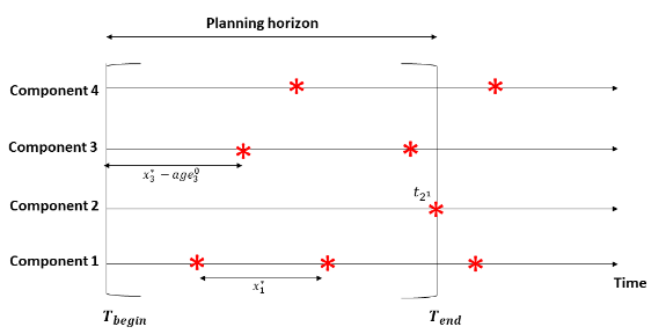

Fig 4. Example of planning horizon for system with 4 components

\subsection{Genetic algorithm}

To identify the best grouping strategy, a Genetic Algorithm (GA) is used in this work. The GA is part of the stochastic optimization algorithms based on the mechanisms of natural selection and genetics. The process starts with an initial population. In a maintenance grouping problem, each candidate solution is encoded as a vector $X$ with $N$ elements corresponding to $N$ maintenance activities. According to $\mathrm{H}$. Vu et $\mathrm{Al}$ (2014) $X$ is structured as:

$$
i^{j} \in G^{k} \rightarrow X\left(i^{j}\right)=k, k \in[1, \ldots,(N-1)]
$$

Where $i^{j}$ the $j^{\text {th }}$ maintenance action if the component $i$ and $k$ the number of the group that contains $i^{j}$. For $N$ maintenance actions, the maximum number of groups is $N-1$.

The initial population is randomly defined and contains $n_{\text {pop }}$ solutions. In each iteration, the GA phases (selection, crossover, mutation and reparation) determines the optimal solution.

During the selection step, the best candidate solutions are selected from population. In this paper, Roulette Wheel Selection is used. The solutions are sorted in ascending order in s groups according to the economic profit. A selection likelihood is associated for each solution proportional to its performance. The candidate solutions (the parent solutions) are used to crossover. A crossover point is randomly chosen to combine selected solutions to generate better solutions in the next generation. In order to generate a new solution, a component of a group is moved to another one. The solutions obtained during the selection and the mutation phases may, sometimes, contains defects for example: empty group, incoherence in actions execution times... During the repair phase, some modifications are made.

- If two maintenance activities $i^{k}$ and $i^{l}$ belong to the group $u$ where $l<k, i^{k}$ is moved, randomly, to another group $v$.

- If $X\left(i^{j}\right)=u$ and $X\left(i^{l}\right)=v$ where $j<l$ and $t_{G} v>t_{G} u$, the maintenance actions are permuted.

- $\quad$ Empty groups are deleted, and the others are renumbered.

\subsection{Mathematic formulation of economic profit}

Let $G^{k}$ be a group of preventive maintenance. The economic profit of a group is divided into three parts as follows:

\section{Setup cost}

The preventive maintenance actions are grouped, only one setup cost is required and is calculated as:

$$
U_{G^{k}}=\left[\operatorname{Card}\left(G^{k}\right)-1\right] . S
$$

Where $\operatorname{Card}\left(G^{k}\right)$ the number of the preventive maintenance activities and $S$ the setup cost.

\section{Penalty cost}

When the maintenance activities are grouped, the maintenance execution time $t_{i}$ is changed to $t^{\prime}{ }_{i j}^{j}=t_{i^{j}}+\Delta t_{i^{j}}$ where $\Delta t_{i^{j}}>-x_{i}{ }^{*}$ (see the figure 4). This modification leads to a penalty cost $\Delta H^{1}{ }_{G}{ }^{k}$ because of reducing service life or increasing likelihood of failure.

Let $h_{i}\left(\Delta t_{i}{ }^{j}\right)$ be the penalty cost when $i^{j}$ is executed at $t_{i j}$ defined as follow:

$$
\begin{gathered}
h_{i}\left(\Delta t_{i^{j}}\right)=E\left[M C_{i}\left(x_{i}^{*}+\Delta t_{i^{j}}\right)\right]-E\left[M C_{i}\left(x_{i}^{*}\right)\right] \\
-\Delta \mathrm{t}_{\mathrm{i}} \mathrm{j} \cdot \mathrm{CA}
\end{gathered}
$$

Where $M C_{i}(x)=C_{i}^{c} \cdot\left(\frac{x}{\alpha}\right)^{\beta}$ the total corrective maintenance cost in the interval time $\left[Q_{i} \beta_{i}\right]$ for the component $i$ and $C A^{*}=\Delta t_{i} j^{j} \frac{C_{i} \beta_{i}}{x_{*}^{*}\left(\beta_{i}-1\right)}$ the average asymptotic cost. The penalty ${ }^{x^{*}}\left(\beta_{i}-1\right)$ of the group $G^{k}$ is computed as:

$$
\Delta \mathrm{H}_{\mathrm{G}^{\mathrm{k}}}^{1}=\sum_{i^{j} \in G^{k}} h\left(\Delta t_{i^{j}}\right)
$$

The optimal execution time of the group $G^{k}$ is found by minimizing $\Delta H_{G^{k}}^{1}$. 


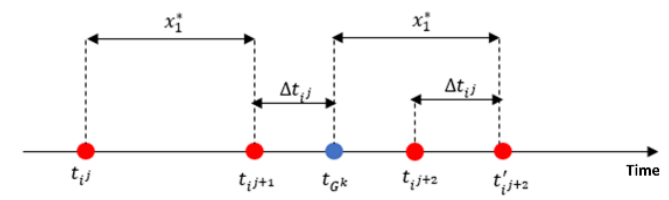

Fig 6. Example of changing execution time of a component $i$

\section{Additional gain due to the structure}

Let $\Delta H^{2}{ }_{G}{ }^{k}$ be the additional gain due to the structure. If $G^{k}$ is a critical group, $\Delta H^{2}{ }_{G^{k}}$ is given in the $\mathrm{Eq}(17)$.

$$
\Delta H_{G^{k}}^{2}=\pi_{G^{k}} \cdot\left(C_{\overline{G^{k}}}-C_{G^{k}}\right)
$$

Where $C_{\overline{G^{k}}}$ is the total planned shutdown cost if the maintenance activities are performed separately:

$$
C_{\overline{G^{k}}}=\sum_{i j \in G^{k}}\left[\pi_{i} \cdot C_{s y s}^{i}+\left(1-\pi_{i}\right) \cdot C_{i}{ }^{l}\right]
$$

$C_{G} k$ is the planned shutdown cost when the maintenance activities are performed together:

$$
C_{G^{k}}=C_{s y s}^{p}
$$
system

Where $C_{s y s}{ }^{p}$ planned shutdown cost of the

\section{Economic profit}

For each group $G^{k}$, the economic profit EP is defined as:

$$
E P\left(G^{k}\right)=U_{G^{k}}-\Delta H^{1}{ }_{G^{k}}-\Delta H^{2}{ }_{G^{k}}
$$

The total economic profit TEP of the grouping structure $S G M$ is the calculated as:

$$
T E P(S G)=\sum_{G^{k} \in S G M} E P\left(G^{k}\right)
$$

A grouping structure is optimal if it maximizes the total economic profit.

$$
S G M_{o p t}=\max _{S G M} T E P(S G M)
$$

\section{Study case}

The grouping strategy is applied to the feedback data of the road markings of the French National Road 4 (NR4) and the Texas's pavement sections introduced in section 2. The failure of one component leads the "shutdown" of the system. Thus, the road infrastructure is presented as a serial system with 4 components. As per the definition of a critical component, all the components are critical, so $\pi_{i}=1, \forall i \in[1,4]$.

It assumed that a road section is composed with pavement and 3 marking lines (Median strip line, Emergency line and skip centre line). For the road markings, for each cluster, the maintenance strategy presented in the section 4 is applicated. The critical age $x_{i}^{*}$ of replacement is defined. The same strategy is used for the pavement's sections. The table 2 is an example of simulated road.

In this case study, the planning horizon started at $T_{\text {Begin }}=0$ : it assumed that the road markings have just been replaced and the pavement is still in a good state (the percent cracks is less than 5\%) and it ends 132 months after $(\mathrm{T}=[0,132])$. Thus, the maintenance activities of the road markings are grouped in this planning horizon.

According to M. Redondin et al (2019), the annual preventive maintenance cost per marking is $C_{p}=940 €$ and the corrective maintenance is supposed $C_{c}=3 * C_{p}$. For the pavement, the exact preventive maintenance cost is still unknown and is assumed equal to $2000 €$ for a section of $152 \mathrm{~m}$. Those parameters can be modified if more cost data are available.

Table 2. Result of application of the EM algorithm and the maintenance strategy

\begin{tabular}{llll}
\hline Components & $\alpha$ & $\beta$ & $x_{i}^{*}$ \\
\hline Pavement & 128.7 & 7.25 & 132 \\
Median strip line & 21.84 & 2.01 & 11 \\
Emergency line & 23.8 & 2.02 & 12 \\
Skip centre line & 30.34 & 6.64 & 19 \\
\hline
\end{tabular}

According to the table 2, the road markings must be replaced each 11,12 , and 19 months. The aim of this section is to identify an optimal time to maintain the road markings simultaneously.

By applying the grouping strategy (presented at section 5), the maintenance activities of the road markings can be grouped every 13 months. Using this policy, the economic profit is about $10.3 \%$.

\section{Conclusions and prospects}

This paper presents a grouping maintenance actions for road infrastructure to optimize the operation of the autonomous vehicles. It assumed that the infrastructure is a serial system with 4 components ( 3 road markings and the pavement). The policy proposed is applied to the feedback data of both French National Road 4 and the American database LTPP. To simplify the study, the road lines and the pavement sections are 
divided into clusters using the Agglomerative Hierarchical Clustering. The datasets are censored; thus, a Weibull analysis is applied to each cluster to estimate the failure dates and the Weibull parameters. Then, an individual maintenance strategy is suggested to each cluster. In order to minimize the maintenance costs and ensure a good state of the road infrastructure, a grouping maintenance actions is proposed using a genetic algorithm.

Future works will consider the dynamic context: changing pavement conditions during the planning horizon, maintenance cost.... The Remaining Useful Lifetime of the pavement will be considered to analyse the pavement state.

\section{Acknowledgment}

VEDECOM and IFSTTAR thank the DIR EST and CEREMA for inspections data of the marking lines of the French National Road 4. Thanks to the Federal Highway Administration for making the LTPP database accessible.

\section{References}

Sasirekha, K., \& Baby, P. (2013) Agglomerative hierarchical clustering algorithm-a., International Journal of Scientific and Research Publications, 83,83

Faddoul R., W Raphael, and Chateauneuf A (2018) Maintenance optimization of series systems subject to reliability constraints, Reliability Engineering \& System Safety 179-188.

McLachlan, G. J., \& Krishnan, T. (2007). The EM algorithm and extensions (Vol. 382). John Wiley \& Sons

Vu H.C, Do P and Barros A. (2015). A stationary grouping maintenance strategy using mean residual life and the Birnbaum importance measure for complex structures. IEEE Transitions on Reliability 65.1: 217-234.

Redondin M, Faul N, Bouillaut L, Daucher D, Same A. (2018). Alternative Weibull analysis for road markings: an EM approach. European Safety and Reliability Conference.

Redondin M, Bouillaut L, Daucher D, Faul N. (2019). A systematic replacement strategy in the context of a strongly censored lifetime. Application to road markings. European Safety and Reliability Conference.

Redondin M, Faul N, Bouillaut L, Daucher D (2017). Temporal clustering of retroreflective marking. European Safety and Reliability Conference.

Zouch M, Yeung T, Castanier B (2009). A ConditionBased Imperfect Maintenance Model with ActionDependent Deterioration. Proceedings of the 2009 Industrial Engineering Research Conference.

Zouch M, Yeung T, Castanier B (2009). A Two-Phase State-Dependent Deterioration Model for
Maintenance Optimization. Naval Research Logistics.

Rausand M and A Hoyland (2004). System Reliability Theory-Models, Statistical Methods, and Applications. Wiley. 